PROCEEDINGS OF THE

AMERICAN MATHEMATICAL SOCIETY

Volume 127, Number 10, Pages 3101-3110

S 0002-9939(99)04834-0

Article electronically published on April 23, 1999

\title{
ON THE MINIMALITY OF POWERS OF MINIMAL $\omega$-BOUNDED ABELIAN GROUPS
}

\author{
DIKRAN DIKRANJAN AND ALBERTO TONOLO \\ (Communicated by Alan Dow)
}

\begin{abstract}
We describe the structure of totally disconnected minimal $\omega$ bounded abelian groups by reducing the description to the case of those of them which are subgroups of powers of the $p$-adic integers $\mathbb{Z}_{p}$. In this case the description is obtained by means of a functorial correspondence, based on Pontryagin duality, between topological and linearly topologized groups introduced by Tonolo. As an application we answer the question (posed in Pseudocompact and countably compact abelian groups: Cartesian products and minimality, Trans. Amer. Math. Soc. 335 (1993), 775-790) when arbitrary powers of minimal $\omega$-bounded abelian groups are minimal. We prove that the positive answer to this question is equivalent to non-existence of measurable cardinals.
\end{abstract}

\section{INTRODUCTION}

All group topologies are assumed to be Hausdorff. We denote by $\hat{G}$ the two-sided (Raïkov) completion of a topological group $G$. A topological group $G$ is precompact if $\hat{G}$ is compact, pseudocompact if every continuous real-valued function on $G$ is bounded, countably compact if every open countable cover of $G$ admits a finite subcover, $\omega$-bounded if every countable subset of $G$ has compact closure, minimal if every continuous group isomorphism $G \rightarrow H$ is open ([Ste]). Every compact group is minimal and $\omega$-bounded, $\omega$-bounded groups are countably compact, countably compact groups are pseudocompact. According to a deep theorem of Prodanov and Stoyanov minimal abelian groups are precompact ([PS]). In this paper we are interested mainly in $\omega$-bounded minimal groups.

The first example of a $\omega$-bounded minimal non-compact group was given by Comfort and Grant $[\mathrm{CG}]$. The group they proposed was non-abelian, zero-dimensional, so in particular totally disconnected. An example of a totally disconnected minimal, $\omega$-bounded non-compact abelian group was given in [DS3, Theorem 1.5]. A connected example was given in [DS2]; it was again non-abelian. In fact, item (b) of the following theorem from [D4] shows that the connected minimal countably compact abelian groups are "frequently" compact. (A cardinal $\alpha$ is measurable if there exists an ultrafilter on $\alpha$ which is closed under countable intersections.)

Received by the editors March 25, 1997 and, in revised form, December 13, 1997.

1991 Mathematics Subject Classification. Primary 54F45, 54D05, 54D30; Secondary 22A05, 22D05, 54D25.

Key words and phrases. Totally disconnected group, connected group, countably compact group, $\omega$-bounded group, minimal group, measurable cardinal.

(C)1999 American Mathematical Society 
Theorem A. Let $G$ be a countably compact abelian group with connected component $c(G)$.

(a) If $c(G)$ is compact, then $G$ is minimal iff $G / c(G)$ is minimal.

(b) If $G$ is minimal and $|c(G)|$ is not measurable, then $c(G)$ is compact.

It should be stressed that the assumption on $|c(G)|$ in item (b) is very weak. In fact, the assumption that there exist no measurable cardinals is known to be consistent with ZFC (actually, $[\mathrm{L}=\mathrm{V}]$ implies measurable cardinals do not exist $[\mathrm{J}]$ ), while it is not known if their existence is consistent with ZFC. Examples of non-compact connected minimal $\omega$-bounded abelian groups of every measurable cardinality are given in [D4].

It was proved in [DS3] that all finite powers of a countably compact minimal abelian group are minimal. The aim of this paper is to answer the following question ([DS3, Question 1.10]) in the case of $\omega$-bounded groups.

Question B. Is $G^{\omega}$ minimal for a countably compact minimal abelian group $G$ ?

In the next theorem we answer positively Question B for minimal $\omega$-bounded totally disconnected abelian groups (Corollary 2) and we show that the answer may be negative in the connected case if measurable cardinals exist (Corollary 3).

Main Theorem. Let $G$ be an $\omega$-bounded minimal abelian group. Then $G^{\omega}$ is minimal iff $c(G)$ is compact.

By this theorem $G^{\omega}$ is minimal for a connected $\omega$-bounded minimal abelian group $G$ iff $G$ is compact.

The background of Question B is the important fact that if $G^{\omega}$ is minimal for a countably compact minimal abelian group, then all powers of $G$ are minimal ([DS3, Corollary 1.9]). This permits one to define the critical power of minimality $\kappa(G)$ of a countably compact minimal abelian group $G$ as 1 if all powers of $G$ are minimal (i.e., $G^{\omega}$ is minimal), otherwise $\kappa(G)$ is the least cardinal $\lambda$ such that $G^{\lambda}$ is not minimal, i.e., $\kappa(G)=\omega$. The critical power of minimality $\kappa(\mathcal{P})$ of a class $\mathcal{P}$ of countably compact minimal abelian groups is defined as $\sup \{\kappa(G): G \in \mathcal{P}\}$. This invariant was introduced and studied for larger classes of minimal abelian groups where it takes more than just two values, 1 and $\omega$, as in the countably compact case (see [St], [D1], [DS1],[DS3] or [DPS, §6.3]). For example, it was shown in [DS3] that under the assumption of Martin's axiom (MA) the critical power of minimality of the class of pseudocompact minimal abelian groups is $2^{\omega}$. Recently ([D6]), a pseudocompact minimal abelian group $G$ was found with $\kappa(G)=\omega_{1}$; moreover, under the assumption of MA, for every cardinal $\lambda$ between $\omega$ and $2^{\omega}$ a pseudocompact minimal abelian group was found with $\kappa(G)=\lambda$. This shows that $\kappa$ provides a good measure to distinguish between pseudocompactness and $\omega$ boundedness within the class of minimal abelian groups (see Corollary 2 and take into account that all pseudocompact minimal abelian groups mentioned above are also totally disconnected).

In view of Theorem A our Main Theorem entails:

Corollary 1. Let $G$ be an $\omega$-bounded minimal abelian group such that $|c(G)|$ is not measurable. Then $\kappa(G)=1$, i.e. all powers of $G$ are minimal.

By the Main Theorem $G^{\omega}$ is minimal for every totally disconnected $\omega$-bounded minimal abelian group $G$. Hence we have: 
Corollary 2. The critical power of minimality of the class of totally disconnected $\omega$-bounded minimal abelian groups is equal to 1.

This corollary reduces the study of the critical power of minimality of the larger class of all $\omega$-bounded minimal abelian groups to that of the connected ones. The proof of the next corollary is given in $\S 2$.

Corollary 3. Let $\alpha$ denote the critical power of minimality of the class of all $\omega$ bounded minimal abelian groups. Then:

(a) $\alpha$ is equal to the critical power of minimality of the class of $\omega$-bounded minimal connected abelian groups,

(b) under the assumption that there exist no measurable cardinals, $\alpha$ is equal to 1 ,

(c) under the assumption that there exist measurable cardinals, $\alpha$ is equal to $\omega$.

In other words, Corollary 3 says that the assertion " $\alpha=1$ " is equivalent to non-existence of measurable cardinals.

The proof of the Main Theorem is obtained from Theorem A in two steps. The first one is a process of localization (described in \$1) that permits one to describe locally the minimality of the powers (Fact 1.4) and reduces the study of totally disconnected $\omega$-bounded minimal abelian groups to the particular case of minimal $\omega$-bounded subgroups of powers of $\mathbb{Z}_{p}$ (Theorem 1.3 and Lemma 1.5). The second step is the following lemma that can be considered as the core of the proof of the Main Theorem.

Main Lemma. Let $\alpha$ be a cardinal number and let $p$ be a prime number. Then for every dense $\omega$-bounded minimal subgroup $G$ of $\mathbb{Z}_{p}^{\alpha}$ there exists $k \in \mathbb{N}$ such that $p^{k} \mathbb{Z}_{p}^{\alpha} \subseteq G$.

The proof of the Main Lemma exploits a functorial correspondence based on Pontryagin duality (see $\S 2$ ). This correspondence was developed by the second author to solve a longstanding problem of Warner on the existence of a finest equivalent linear module topology ([T1]) and to study minimality and total minimality in topological modules covered by compact submodules ([T2]). Through this correspondence (see Lemma 2.1) the Main Lemma is translated in an equivalent question on linear topologies on direct sums of the Prüfer group $\mathbb{Z}\left(p^{\infty}\right)$.

The proof of the next corollary of the Main Lemma concerning the structure of totally disconnected $\omega$-bounded minimal abelian groups is given in $\S 2$.

Corollary 4. Let $G$ be a totally disconnected $\omega$-bounded minimal abelian group. Then there exists a compact subgroup $N$ of $G$ such that the quotient group $G / N$ is a direct product (provided with the product topology) of $\omega$-bounded torsion minimal groups $G_{p}$.

We denote by $\mathbb{N}$ and $\mathbb{P}$ the sets of naturals and primes, respectively, by $\mathbb{Z}$ the integers, by $\mathbb{R}$ the reals, by $\mathbb{Z}_{p}$ the $p$-adic integers $(p \in \mathbb{P})$. For undefined symbols or notions see $[\mathrm{E}],[\mathrm{HR}]$ or $[\mathrm{J}]$.

\section{The LOCALIZATION - QUASI- $p$-TORSION ELEMENTS}

The following minimality criterion of Banaschewski-Prodanov-Stephenson is very important. A subgroup $H$ of a topological group $G$ is essential if for every nontrivial closed normal subgroup $N$ of $G$ the intersection $H \cap N$ is non-trivial. 
1.1 Theorem ([B], $[\mathrm{P}]$, $[\mathrm{Ste}])$. Let $G$ be a topological group and $H$ be a dense subgroup of $G$. Then $H$ is minimal iff $G$ is minimal and $H$ is essential in $G$.

1.2 Definition ([St]). For $p \in \mathbb{P}$ and a topological abelian group $G$ an element $x \in G$ is quasi-p-torsion if $\langle x\rangle$ is either a finite $p$-group or equipped with the induced topology is isomorphic to $\left(\mathbb{Z}, \tau_{p}\right)$, where $\tau_{p}$ is the $p$-adic topology of $\mathbb{Z}$.

The set $t d_{p}(G)$ of all quasi-p-torsion elements of $G$ is a subgroup of $G$ ([DPS, Chap.4]).

Let us recall that $\omega$-bounded groups are precompact and quasi- $p$-torsion precompact abelian groups are totally disconnected ([D2]).

We show next that in analogy with the compact case, every $\omega$-bounded minimal totally disconnected abelian group $G$ decomposes into a direct product of its quasi $p$ torsion subgroups $t d_{p}(G)$. This settles the classification of the totally disconnected minimal $\omega$-bounded abelian groups by reduction to the case of quasi- $p$-torsion minimal $\omega$-bounded abelian groups. The counterpart of the following theorem for the case of countably compact groups is given in [D4]. Here we give a proof in the $\omega$-bounded case which is different, entirely based on the corresponding property of the compact totally disconnected abelian groups.

1.3 Theorem. Let $G$ be a $\omega$-bounded totally disconnected abelian group. Then for each $p \in \mathbb{P}$ the subgroup $t_{p}(G)$ is closed and

$$
G=\prod_{p \in \mathbb{P}} t d_{p}(G)
$$

with the product topology. Moreover, $G$ is minimal iff each $t d_{p}(G)$ is minimal.

Proof. It is known that for a compact totally disconnected abelian group $K$ the subgroup $t d_{p}(K)$ is closed for each $p \in \mathbb{P}$ and $K=\prod_{p \in \mathbb{P}} t d_{p}(K)$ with the product topology (see [DPS, Example 4.1.3 (a)]). Applying this fact to the compact group $K=\hat{G}$ we get $\hat{G}=\prod_{p \in \mathbb{P}} t d_{p}(\hat{G})$ with the product topology. Moreover, for each $p \in \mathbb{P}$ the subgroup $t d_{p}(G)$ of $G$ is closed as $t d_{p}(G)=G \cap t d_{p}(\hat{G})$. To prove (1) let us first observe that $G$, being $\omega$-bounded, is covered by compact subgroups, and for every one of them, say $H$, we have $H=\prod_{p \in \mathbb{P}} t d_{p}(H) \subseteq \prod_{p \in \mathbb{P}} t d_{p}(G)$ since $t d_{p}(H) \subseteq t d_{p}(G)$. This proves the inclusion $\subseteq$ in (1). To prove the other inclusion observe that $\oplus t d_{p}(G) \subseteq G$, hence an arbitrary element $x=\left(x_{p}\right) \in \prod_{p \in \mathbb{P}} t d_{p}(G)$ can be presented as an element of the closure (taken in $\hat{G}$ ) of a countably generated subgroup of $G$. Since countably generated subgroups of $G$ are contained in compact subgroups of $G$, this gives $x \in G$. So the inclusion $\supseteq$ in (1) is proved as well.

The last assertion follows from Corollary 6.1.3 of [DPS] (see also [D1]).

For a topological abelian group $G$, a prime number $p$ and $k \in \mathbb{N}, G$ is strongly $p$-dense (of degree $k$ ) iff there exists $k \in \mathbb{N}$ with $p^{k} t d_{p}(\hat{G}) \subseteq G$ (see [D1, Definition 1] or [DS1, p. 586]). For a quasi- $p$-torsion abelian group $G$ this condition simplifies to $p^{k} \hat{G} \subseteq G$. Non-compact strongly $p$-dense quasi- $p$-torsion $\omega$-bounded minimal abelian groups exist in profusion [DS3, Corollary 1.6].

The following fact explains the relation of strong $p$-density to minimality of powers.

1.4 Fact ([St], [D1, Corollaire 7]). Let $G$ be a minimal abelian group. Then all powers of $G$ are minimal iff $G$ is strongly $p$-dense for every prime number $p$. 
It follows from the minimality criterion, that the completion $\hat{G}$ of a torsion-free minimal abelian group $G$ is torsion-free. Since torsion-free quasi-p-torsion compact abelian groups are of the form $\mathbb{Z}_{p}^{\alpha}$, the torsion-free quasi- $p$-torsion $\omega$-bounded minimal abelian groups are subgroups of powers of $\mathbb{Z}_{p}$. Hence our Main Lemma says that a torsion-free quasi-p-torsion $\omega$-bounded minimal abelian group must be strongly p-dense. Actually, by means of the localization developed in Theorem 1.3 and Lemma 1.5 we prove more: every totally disconnected $\omega$-bounded minimal abelian group is strongly $p$-dense for every prime $p$.

Now we reduce the study of quasi-p-torsion $\omega$-bounded minimal abelian groups to the study of those which are torsion-free, i.e., subgroups of powers of $\mathbb{Z}_{p}$.

1.5 Lemma. Fix a prime $p$. Let $G$ be a minimal quasi-p-torsion $\omega$-bounded abelian group. Then, with $\alpha=w(G)$, there exist a dense minimal $\omega$-bounded subgroup $G_{1}$ of $\mathbb{Z}_{p}^{\alpha}$ and a compact subgroup $N$ of $G_{1}$ such that:

(a) the quotient group $G_{1} / N$ is isomorphic to $G$;

(b) $G$ and $G_{1}$ have the same degree of strong p-density.

Proof. Since $\hat{G}$ is a compact $\mathbb{Z}_{p}$-module, there exists a continuous surjective homomorphism $f: K=\mathbb{Z}_{p}^{\alpha} \rightarrow \hat{G}$ (see [DPS]). Then $G_{1}=f^{-1}(G)$ is a dense $\omega$-bounded subgroup of $K$ ([DS2, Lemma 2.1]). Minimality of $G_{1}$ follows from Theorem 1.1 and the fact that $G_{1}$ is essential in $K$.

Set $N=\operatorname{ker} f$; then $G \cong G_{1} / N$ by the Sulley-Grant lemma ([DPS, Lemma 4.3.2]). This proves (a).

To prove (b) note that $p^{k} \hat{G} \subseteq G$ is equivalent to $p^{k} \mathbb{Z}_{p}^{\alpha} \subseteq G_{1}$.

\section{Proof of the MAIN RESUlts}

We give briefly an outline of the functorial correspondence between precompact groups covered by their compact subgroups and linearly topologized groups developed in $[\mathrm{T} 1]$ and $[\mathrm{T} 2]$. Let $\mathbb{T}$ be the circle group $\mathbb{R} / \mathbb{Z}$ endowed with the usual topology. The Pontryagin duality associates to each compact group $K$ the abstract group $K^{*}=\operatorname{Chom}(K, \mathbb{T})$ of continuous homomorphisms of $K$ in $\mathbb{T}$, to each abstract group $X$ the group $X^{*}=\operatorname{Hom}(X, \mathbb{T})$ of homomorphisms of $X$ in $\mathbb{T}$, endowed with the pointwise-convergence topology, and to each homomorphism, of compact or abstract groups, its transpose.

Let $G$ be a precompact abelian group such that every element of $G$ is contained in a compact subgroup of $G$. Then the family $\mathcal{M}=\left\{M_{\lambda}: \lambda \in \Lambda\right\}$ of compact separable subgroups of $G$ (i.e., the subgroups which have a dense countable subset) is directed with respect to inclusion and $G=\bigcup_{\lambda} M_{\lambda}$. Let $X$ be the Pontryagin dual of the compact completion $\hat{G}$ of $G$. For each $M \in \mathcal{M}$, consider the annihilator $\operatorname{Ann}(M)=\{\xi \in X: \xi(M)=0\}$. The family $\{\operatorname{Ann}(M): M \in \mathcal{M}\}$ is a filter base of subgroups of $X$; hence it defines a linear topology $\tau$ on $X$, i.e. a group topology which has a base of neighbourhoods of zero consisting of subgroups.

The idea is to express properties of $G$ in terms of properties of $(X, \tau)$.

Lemma 2.1. Let $G, X$ and $\tau$ be as above.

(i) The group $G$ is strongly $p$-dense with degree $k$ if and only if for every subgroup $L$ of $X$ with $X / L \leq \mathbb{Z}\left(p^{\infty}\right)$ the subgroup $\left(L: p^{k}\right)=\left\{x \in X: p^{k} x \in L\right\}$ is $\tau$-closed.

(ii) The group $G$ is minimal if and only if $(X, \tau)$ has no dense proper subgroups. 
(iii) The group $G$ is $\omega$-bounded if and only if the space $(X, \tau)$ is a P-space, i.e., countable intersections of $\tau$-open sets are $\tau$-open.

Proof. (i) Consider the algebraic monomorphism $X / L \rightarrow \mathbb{Z}\left(p^{\infty}\right)$. The Pontryagin duality produces a surjective continuous homomorphism $\psi: \mathbb{Z}_{p} \rightarrow \operatorname{Ann} L$. The element $x=\psi(1)$ belongs to $t d_{p}(\hat{G})$ and $(x)=\overline{\langle x\rangle}=\operatorname{Ann} L$. If $G$ is strongly $p$-dense with degree $k$, then $p^{k} x \in G$, hence $\left(p^{k} x\right)=p^{k}(x) \leq G$. Now $p^{k}(x)$ is a separable compact subgroup of $G$, hence

$$
\operatorname{Ann} p^{k}(x)=\left(\operatorname{Ann}(x): p^{k}\right)=\left(L: p^{k}\right)
$$

is $\tau$-open, hence $\tau$-closed. Conversely, given $x \in t d_{p}(\hat{G})$, let us consider the surjective continuous epimorphism $\psi: \mathbb{Z}_{p} \rightarrow(x)$, defined by $\psi(1)=x$. The Pontryagin duality produces the monomorphism

$$
(x)^{*}=X / \operatorname{Ann}(x) \rightarrow \mathbb{Z}\left(p^{\infty}\right) .
$$

By hypothesis $\left(\operatorname{Ann}(x): p^{k}\right)=\operatorname{Ann} p^{k}(x)$ is $\tau$-closed; since $X /\left[\operatorname{Ann} p^{k}(x)\right] \leq \mathbb{Z}\left(p^{\infty}\right)$ is Hausdorff and $\mathbb{Z}\left(p^{\infty}\right)$ is finitely cogenerated, $\operatorname{Ann} p^{k}(x)$ is also $\tau$-open. Then $p^{k}(x)$ is contained in a (separable compact) subgroup of $G$; in particular $p^{k} x$ belongs to G.

(ii) Also the family of annihilators of all compact subgroups of $G$ defines a linear topology $\sigma$ on $X$, clearly finer than $\tau$. By Proposition 2.2 (see the proof of point i)) of [T2], it is possible to verify that $\sigma$ and $\tau$ are equivalent, i.e. they determine the same closed subgroups of $X$. In [T2, Theorem 3.1] the second author has proved that $G$ is essential in $\hat{G}$ if and only if $(X, \sigma)$ has no dense proper subgroups. Since $\tau$ and $\sigma$ are equivalent, $G$ is essential in $\hat{G}$ if and only if $(X, \tau)$ has no dense proper subgroups. Then we conclude by Theorem 1.1.

(iii) Clearly, the topology $\tau$ is a $P$-topology if and only if the family $\mathcal{M}$ of compact separable subgroups of $G$ is $\omega$-directed, i.e., for every countable family $\left\{L_{i}: i \in \mathbb{N}\right\}$ of $\mathcal{M}$ there exists $L \in \mathcal{M}$ containing all $L_{i}$. Let us prove that this happens if and only if the group $G$ is $\omega$-bounded. Let $G$ be $\omega$-bounded and let $\left\{L_{i}: i \in \mathbb{N}\right\}$ be a family in $\mathcal{M}$. For each $i \in \mathbb{N}$, denote by $J_{i}$ a countable dense subset of $L_{i}$. Then the set $\bigcup_{i \in \mathbb{N}} J_{i}$ is countable and $L=\overline{\bigcup_{i \in \mathbb{N}} J_{i}} \in \mathcal{M}$ contains every $L_{i}$. Conversely, suppose the family $\mathcal{M}$ is $\omega$-directed. Let $J$ be a countable subset of $G$. Then $\overline{\langle j\rangle} \in \mathcal{M}$ for each $j \in J$. By our hypothesis there exists a compact subgroup $L \in \mathcal{M}$ that contains $\overline{\langle j\rangle}$ for every $j \in J$. Hence the closure of $J$ in $G$ is compact.

Proof of the Main Lemma. Let $G$ be a dense $\omega$-bounded minimal subgroup of $\mathbb{Z}_{p}^{\alpha}$. Then by [DS3, Lemma 3.1] every element of $G$ is contained in a compact subgroup of $G$. The Pontryagin dual of $\mathbb{Z}_{p}^{\alpha}$ is $\mathbb{Z}\left(p^{\infty}\right)^{(\alpha)}$, so that by Lemma 2.1, after dualizing, our Main Lemma takes the following form. Consider $\mathbb{Z}\left(p^{\infty}\right)^{(\alpha)}$ equipped with a linear Hausdorff topology $\tau$ satisfying the following conditions:

i) there exist no proper $\tau$-dense subgroups of $\left(\mathbb{Z}\left(p^{\infty}\right)^{(\alpha)}, \tau\right)$;

ii) countable intersections of $\tau$-open sets are $\tau$-open.

For every subgroup $L$ of $\mathbb{Z}\left(p^{\infty}\right)^{(\alpha)}$ with $\left[\mathbb{Z}\left(p^{\infty}\right)^{(\alpha)}\right] / L \cong \mathbb{Z}\left(p^{\infty}\right)$ there exists $n=$ $n_{L} \in \mathbb{N}$ such that $\left(L: p^{n}\right)$ is $\tau$-open. This is the number $n$ determined by the $\tau$-closure $\bar{L}$ of $L$ : by (i) it cannot be the whole $\mathbb{Z}\left(p^{\infty}\right)^{(\alpha)}$, so that $\bar{L} / L$ must be isomorphic to a proper subgroup, say $\mathbb{Z}\left(p^{n}\right)$, of Prüfer's group. We have to prove that the number $n$ can be chosen independently of $L$. 
Let us denote by $H_{n}$ the unique subgroup of $\mathbb{Z}\left(p^{\infty}\right)$ of order $p^{n}$. Suppose there exist subgroups $L_{1}, \ldots, L_{n}, \ldots$ of $\mathbb{Z}\left(p^{\infty}\right)^{(\alpha)}$ such that $\left[\mathbb{Z}\left(p^{\infty}\right)^{(\alpha)}\right] / L_{i} \cong \mathbb{Z}\left(p^{\infty}\right)$ and such that the orders $\left|\overline{L_{i}} / L_{i}\right|=p^{n_{i}}$ form a strictly increasing sequence of natural numbers. It is not restrictive to suppose $\left|\overline{L_{i}} / L_{i}\right|=p^{i}$. The subgroups $L_{i}$ are kernels of morphisms

$$
f_{i}: \mathbb{Z}\left(p^{\infty}\right)^{(\alpha)} \rightarrow \mathbb{Z}\left(p^{\infty}\right)
$$

Since

$$
\operatorname{Hom}\left(\mathbb{Z}\left(p^{\infty}\right)^{(\alpha)}, \mathbb{Z}\left(p^{\infty}\right)\right) \cong\left[\operatorname{Hom}\left(\mathbb{Z}\left(p^{\infty}\right), \mathbb{Z}\left(p^{\infty}\right)\right)\right]^{\alpha}
$$

and

$$
\operatorname{Hom}\left(\mathbb{Z}\left(p^{\infty}\right), \mathbb{Z}\left(p^{\infty}\right)\right) \cong \mathbb{Z}_{p}
$$

for each $f_{i}$ we have some $\mu_{i}=\left(\mu_{i, x}\right)_{x \in \alpha}$ belonging to $\mathbb{Z}_{p}^{\alpha}$ such that

$$
f_{i}\left(\left(a_{x}\right)_{x \in \alpha}\right)=\sum_{x \in \alpha} \mu_{i, x} a_{x} .
$$

Since the only Hausdorff linear topology on $\mathbb{Z}\left(p^{\infty}\right)$ is the discrete one, every $\overline{L_{i}}$ is open. Then, by the hypothesis ii) on $\tau, W=\bigcap_{i \in \mathbb{N}} \overline{L_{i}}$ is an open subgroup of $\mathbb{Z}\left(p^{\infty}\right)^{(\alpha)}$. Clearly, for each $i \in \mathbb{N}$ and each open subgroup $U \leq W$, we have $L_{i}+U=\overline{L_{i}}$. Since $\overline{L_{i}} / L_{i} \cong H_{i}$, for each open subgroup $U \leq W$

$$
f_{i}\left(\overline{L_{i}}\right)=f_{i}\left(L_{i}+U\right)=f_{i}\left(L_{i}\right)+f_{i}(U)=f_{i}(U)=H_{i}
$$

hold. For $u \in \mathbb{Z}\left(p^{\infty}\right)^{(\alpha)}$ denote by $|u|$ the order of $u$. For each $i \in \mathbb{N}$, there exists a natural number $\nu(i)>0$ such that for every open subgroup $U$ there exists $u \in U$ with $|u|<\nu(i)$ such that $f_{i}(u)$ generates $H_{i}$. Otherwise, for each $n \in \mathbb{N}$ there would be an open subgroup $U_{n}$ such that for no element $u \in U_{n}$ with $|u|<n$, does $f_{i}(u)$ generate $H_{i}$; then, $U_{\infty}=\bigcap_{n \in \mathbb{N}} U_{n}$ would be an open subgroup with $f_{i}\left(U_{\infty}\right)$ properly contained in $H_{i}$ : a contradiction.

Now for $i \in \mathbb{N}$ define $\psi(i)=\max \left\{\nu\left(i^{2}\right), i+1\right\}$. Since

$$
\psi(1)<\psi^{2}(1)<\cdots<\psi^{j}(1)<\ldots,
$$

the series

$$
\mu_{1}+\sum_{j \geq 1} p^{\psi^{j}(1)} \mu_{\left[\psi^{j}(1)\right]^{2}}
$$

defines an element $\bar{\mu} \in \mathbb{Z}_{p}^{\alpha}$.

Let $L$ be the kernel of the morphism $f$ associated to $\bar{\mu}$, i.e.,

$$
f: \mathbb{Z}\left(p^{\infty}\right)^{(\alpha)} \rightarrow \mathbb{Z}\left(p^{\infty}\right), \quad\left(a_{x}\right)_{x \in \alpha} \mapsto \sum_{x} \bar{\mu}_{x} a_{x} .
$$

Let us prove that $L$ is a proper dense subgroup of $\mathbb{Z}\left(p^{\infty}\right)^{(\alpha)}$ contradicting the hypotheses.

For every $U \leq W$ and every $j \in \mathbb{N}$ we have

$$
p^{\psi^{j}(1)} f_{\left[\psi^{j}(1)\right]^{2}}(U)=p^{\psi^{j}(1)} H_{\left[\psi^{j}(1)\right]^{2}}=H_{\left[\psi^{j}(1)\right]^{2}-\psi^{j}(1)} .
$$

Moreover, there exists $u^{(j)} \in U$ with $\left|u^{(j)}\right|<\nu\left(\left[\psi^{j}(1)\right]^{2}\right)$, such that $p^{\psi^{j}(1)} f_{\left[\psi^{j}(1)\right]^{2}}\left(u^{(j)}\right)$ generates $H_{\left[\psi^{j}(1)\right]^{2}-\psi^{j}(1)}$. Now we prove that also $f\left(u^{(j)}\right)$ generates $H_{\left[\psi^{j}(1)\right]^{2}-\psi^{j}(1)}$. 
Indeed,

$$
\begin{gathered}
f\left(u^{(j)}\right)=\sum_{x} \bar{\mu}_{x} u_{x}^{(j)}=\sum_{x}\left(\mu_{1, x}+\sum_{i \geq 1} p^{\psi^{i}(1)} \mu_{\left[\psi^{i}(1)\right]^{2}, x}\right) u_{x}^{(j)} \\
=\left[\sum_{x}\left(\mu_{1, x}+\sum_{i=1}^{j-1}\left(p^{\psi^{i}(1)} \mu_{\left[\psi^{i}(1)\right]^{2}, x}\right) u_{x}^{(j)}\right)\right]+\sum_{x}\left(p^{\psi^{j}(1)} \mu_{\left[\psi^{j}(1)\right]^{2}, x}\right) u_{x}^{(j)} \\
+\left[\sum_{x}\left(\sum_{i \geq j+1}\left(p^{\psi^{i}(1)} \mu_{\left[\psi^{i}(1)\right]^{2}, x}\right) u_{x}^{(j)}\right)\right]
\end{gathered}
$$

The first summand is contained in $H_{\left[\psi^{j-1}(1)\right]^{2}-\psi^{j-1}(1)}$, the second one generates $H_{\left[\psi^{j}(1)\right]^{2}-\psi^{j}(1)}$ and the third one vanishes since for each $l \geq 1$ we have

$$
\psi^{j+l}(1)=\psi^{l}\left(\psi^{j}(1)\right)>\psi\left(\psi^{j}(1)\right) \geq \nu\left(\left[\psi^{j}(1)\right]^{2}\right)>\left|u^{(j)}\right| .
$$

By (2) the sequence $\left[\psi^{j}(1)\right]^{2}-\psi^{j}(1), j \in \mathbb{N}$, is strictly increasing (note that the function $x \mapsto x^{2}-x$ is strictly increasing for $\left.x>1\right)$. Hence the family $\left\{f\left(u^{(j)}\right)\right.$ : $j \in \mathbb{N}\}$ generates $\mathbb{Z}\left(p^{\infty}\right)$.

We have proved in this way that for each open subgroup $U$ contained in $W$, $f(U)=\mathbb{Z}\left(p^{\infty}\right)$. Therefore $L$ is dense - a contradiction.

Proof of Main Theorem. Since $C=c(G)$ is connected, its compact completion $\hat{C}$ is connected, hence divisible ([HR]). Now the minimality of $G^{\omega}$ yields that $C^{\omega}$ is minimal, as a closed subgroup of the minimal group $G^{\omega}$. By [DS3, Corollary 1.9] this gives $\kappa(C)=1$, hence, by Fact 1.4, $C$ is strongly $p$-dense for every prime $p$. The divisibility of $\hat{C}$ yields that $t d_{p}(\hat{C})$ is divisible for every prime $p$ ([DPS, Proposition 4.1.2]). Hence $t d_{p}(\hat{C}) \subseteq C$ for every prime $p$. Then $C$ is totally minimal ([DPS, Theorem 4.3.7]). Since $C$ is also countably compact, it follows from [DS2] that $C$ is compact.

Suppose now that $C$ is compact and let $\alpha$ be a cardinal. The quotient $H=G / C$ is totally disconnected and $\omega$-bounded; moreover $c\left(G^{\alpha}\right)=C^{\alpha}$ and $H^{\alpha} \cong G^{\alpha} / c\left(G^{\alpha}\right)$. By Theorem A, $H=G / C$ is a minimal group. If we prove that $H^{\alpha}$ is minimal, Theorem A will imply that the group $G^{\alpha}$ is minimal as well. Hence it suffices to consider only the totally disconnected case.

From now on we assume $G$ is an $\omega$-bounded totally disconnected minimal abelian group. According to Fact 1.4 the minimality of all powers $G^{\alpha}$ for such group $G$ follows from the strong $p$-density of $G$ for every prime $p$. By Theorem 1.3 the subgroup $t d_{p}(G)$ of quasi-p-torsion elements of $G$ is still minimal and $\omega$-bounded, moreover, $\overline{t d_{p}(G)}$ coincides with $t d_{p}(\hat{G})$. Therefore, strong $p$-density for $G$ is equivalent with strong $p$-density for the quasi- $p$-torsion group $t d_{p}(G)$. By Lemma 1.5 it suffices to consider quasi- $p$-torsion groups that are subgroups of powers of $\mathbb{Z}_{p}$. In such a case the strong $p$-density of $G$ was established in our Main Lemma.

Proof of Corollary 3. (a) If $G^{\omega}$ is not minimal for some $\omega$-bounded abelian group $G$, then $c(G)$ is not compact by Main Theorem. Hence $c(G)$ is a connected $\omega$ bounded minimal abelian group that is not compact, hence $c(G)^{\omega}$ is not minimal again by Main Theorem.

(b) Follows from Corollary 2 and Theorem A. 
(c) For the connected $\omega$-bounded minimal abelian group $G$ of measurable weight constructed in [D4] the power $G^{\omega}$ is not minimal according to Main Theorem since $G$ is not compact.

Proof of Corollary 4. Let $f: K=\prod_{p \in \mathbb{P}} \mathbb{Z}_{p}^{\sigma} \rightarrow \hat{G}$ be a continuous surjective homomorphism (see [DPS]). Then $H=f^{-1}(G)$ is a dense $\omega$-bounded minimal subgroup of $K$. Set $K_{p}=\mathbb{Z}_{p}^{\sigma}$; then for each $p$ the subgroup $H_{p}=H \cap K_{p}$ of $K_{p}$ is an essential $\omega$-bounded subgroup of $K_{p}$. So by the Main Theorem there exists a natural $k_{p}$ such that $p^{k_{p}} K_{p} \subseteq H_{p}$ for each $p$. Then for $N_{1}=\prod p^{k_{p}+1} K_{p}$ and $N=f\left(N_{1}\right)$ obviously $G / N \cong K / N_{1} \cong \prod_{p} K_{p} /\left(N_{1} \cap K_{p}\right)$ and each quotient $K_{p} /\left(N_{1} \cap K_{p}\right)$ is obviously a compact torsion abelian group. Moreover, every $f\left(H_{p}\right)$ is minimal as a dense subgroup of $L_{p}=K_{p} / p^{k_{p}+1} K_{p} \cong \mathbb{Z}\left(p^{k_{p}+1}\right)^{\sigma}$ containing the socle $f\left(p^{k_{p}} K_{p}\right)$ of $L_{p}$.

It follows from this corollary and Theorem A that the connected component $C$ of an $\omega$-bounded minimal abelian group $G$ of non-measurable cardinality is compact and contained in a compact subgroup $N$ of $G$ such that the quotient $G / N$ is a direct product (provided with the product topology) of $\omega$-bounded torsion minimal groups.

We finish by showing a possible way of extension of our Main Lemma to the countably compact case. The following result on approximation by large $\omega$-bounded subgroups was proved in [D4]:

2.2 Theorem. Every minimal torsion-free connected countably compact abelian group $G$ contains a minimal connected $\omega$-bounded subgroup $G_{\omega}$ such that its closure $\overline{G_{\omega}}$ is a $G_{\delta}$-subgroup of $G$.

We do not know if one can replace "connected" by "totally disconnected" in this theorem. In case the answer is positive, then the Main Lemma implies that for every prime $p$, a torsion-free quasi-p-torsion countably compact minimal abelian group $G$ must be strongly $p$-dense. In fact, $\hat{G} \cong \mathbb{Z}_{p}^{\alpha}$. Let $O_{n}, n \in \mathbb{N}$, be open subgroups of $\hat{G}$ such that for $V=\bigcap_{n} O_{n}$ we have $\overline{G_{\omega}}=G \cap V$. Since $G$ is $G_{\delta}$-dense in $\hat{G}$ (as $G$ is pseudocompact, cf. [CR]), it follows that $\overline{G_{\omega}}$ is dense in $V$, i.e., $V$ is the completion of $G_{\omega}$. By the Main Lemma there exists $k \in \mathbb{N}$ such that $p^{k} V \subseteq G_{\omega} \subseteq G$. By the definition of the Tychonov topology of $\mathbb{Z}_{p}^{\alpha}=\hat{G}$, there exists a countable $D \subseteq \alpha$, such that $U=\{0\} \times \mathbb{Z}_{p}^{\alpha \backslash D} \subseteq V$. Since $U \subseteq V$, obviously $p^{k} U \subseteq G$. Now consider the closed subgroup $G_{1}:=G \cap \mathbb{Z}_{p}^{D}$ of $G$. By the minimality of $G$, it is essential in $\mathbb{Z}_{p}^{D}$. Since $G_{1}$ is compact (as a metrizable subgroup of a countably compact group), it is closed in $\mathbb{Z}_{p}^{D}$ as well. Now the essentiality means that the quotient group $\mathbb{Z}_{p}^{D} / G_{1}$ is torsion. Since it is compact abelian, it must have a finite exponent $p^{m}$. Thus $p^{m} \mathbb{Z}_{p}^{D} \subseteq G$. Then, with $n=\max \{k, m\}$, we get $p^{n} \hat{G} \subseteq G$.

\section{REFERENCES}

[B] B.Banaschewski, Minimal topological algebras, Math. Ann. 211 (1974), 107-114. MR 50:9988

[CG] W. W. Comfort and D. Grant, Cardinal invariants, pseudocompactness and minimality: Some recent advances in the topological theory of topological groups, Topology Proc. 6 (1981), 227-265. MR 84b:54009

[CR] W. Comfort and K. Ross, Pseudocompactness and uniform continuity in topological groups, Pacific J. Math. 16 (1966), 483-496. MR 34:7699 
[D1] D. Dikranjan, Sur la minimalité des produits de groupes topologiques abéliens, C. R. Acad. Sci. Paris 299 Série I (1984), 303-306. MR 86a:22001

[D2] D. Dikranjan, Zero-dimensionality of some pseudocompact groups, Proc. Amer. Math. Soc. 120 n. 4 (1994), 1299-1308. MR 94f:54077

[D3] D. Dikranjan, Compactness and connectedness in topological groups, Topology Appl., 84 (1998), 227-252. CMP 98:10

[D4] D. Dikranjan, The structure of minimal countably compact abelian groups, Preprint.

[D5] D. Dikranjan, Recent advances in minimal topological groups, Topology Appl., 85 (1998), 53-91. CMP 98:11

[D6] D. Dikranjan, p-adic numbers and minimality of powers, Preprint.

[DPS] D. Dikranjan, I. Prodanov and L. Stoyanov, Topological groups: characters, dualities and minimal group topologies, Monographs and Textbooks in Pure and Applied Mathematics, Vol. 130, Marcel Dekker Inc., New York-Basel, 1990. MR 91e:22001

[DS1] D. Dikranjan and D. Shakhmatov, Products of minimal abelian groups, Math. Zeit. 204 (1990), 583-603. MR 91i:22002

[DS2] D. Dikranjan and D. Shakhmatov, Compact-like totally dense subgroups of compact groups, Proc. Amer. Math. Soc. 114 (1992), 1119-1129. MR 92g:22009

[DS3] D. Dikranjan and D. Shakhmatov, Pseudocompact and countably compact abelian groups: Cartesian products and minimality, Trans. Amer. Math. Soc. 335 (1993), 775-790. MR 93d:22001

[E] R. Engelking, General Topology, 2nd edition, Heldermann Verlag, Berlin, 1989. MR 91c:54001

[HR] E. Hewitt and K. A. Ross, Abstract harmonic analysis. Vol. 1, 2nd edition, Springer-Verlag, 1979. MR 81k:43001

[J] T. Jech, Set Theory, Academic Press, New York, 1978. MR 80a:03062

[P] I. Prodanov, Precompact minimal group topologies and p-adic numbers, Annuaire Univ. Sofia Fac. Math. Méc. 66 (1971/72), 249-266. MR 54:449

[PS] I. Prodanov and L.Stoyanov, Every minimal abelian group is precompact, C. R. Acad. Bulgare Sci. 37 (1984), 23-26. MR 85k:22007

[Ste] R. M. Stephenson, Jr., Minimal topological groups, Math. Ann. 192 (1971), 193-195. MR 44:4141

[St] L. Stoyanov, Weak periodicity and minimality of topological groups, Annuaire Univ. Sofia Fac. Math. Méc. 73 (1978/79), 155-167. MR 89a:22003

[T1] A. Tonolo, On the existence of a finest equivalent linear topology, Comm. Algebra 20(2) (1992), 437-455, Erratum ibid. 22(6), (1994), 2317. CMP 94:10

[T2] A. Tonolo, On a class of minimal topological modules, in: A. Facchini and C. Menini ed., Abelian Groups and Modules, Proc. of the Padova Conference, Padova, Italy, June 23-July 1, 1994, Mathematics and its Applications, 343 Kluwer Academic Publishers, DordrechtBoston-London, The Netherlands (1995), 459-466. MR 97a:16090

Dipartimento di Matematica e Informatica, Udine University, via delle Scienze 206 (LOCAlitÀ RizZI), 33100 Udine, ItAly

E-mail address: dikranja@dimi.uniud.it

Dipartimento di Matematica Pura ed Applicata, Padova University, Via Belzoni 7, 35131 PADOVA, ITALY

E-mail address: tonolo@math.unipd.it 